\title{
CONTRIBUIÇÕES PARA O DEBATE SOBRE A ESCOLA PÚBLICA: $O$ ÍNDICE DE DESENVOLVIMENTO DA EDUCAÇÃO BÁSICA (IDEB)
}

\author{
Fábio Mariano Da Paz ${ }^{1}$, Hélia Sônia Raphae ${ }^{2}$ \\ ${ }^{1}$ Doutorando pelo Programa de Pós-Graduação em Educação da Faculdade de Ciências e Tecnologia, Unesp - Campus de e \\ Presidente Prudente - SP. ${ }^{2}$ Docente atualmente aposentada do Programa de Pós-Graduação em Educação da Faculdade de Ciências \\ e Tecnologia, UNESP. E-mail: fmpaz@ig.com.br
}

\begin{abstract}
RESUMO
Este estudo apresenta parte dos resultados obtidos na pesquisa de mestrado intitulada "O Índice de Desenvolvimento da Educação Básica (IDEB): um estudo do município de Santa Fé do Sul/SP." Tem por objetivo abordar a operacionalização do Plano de Desenvolvimento da Educação (PDE) e sua culminância no IDEB, analisando globalmente seus fundamentos, problemas e perspectivas. Pretende ainda, partindo de dados provenientes de pesquisa documental, entrevistas e questionários aplicados, refletir sobre os impactos dos rankings produzidos em função do IDEB, bem como as práticas cada vez mais correntes de responsabilização das escolas. Para proceder à pesquisa no município de Santa Fé do Sul foi preciso elucidar quais mecanismos desencadearam elevado aumento no IDEB 2007, em comparação a 2005. As análises empreendidas levaram à constatação de falhas evidentes na consolidação do IDEB 2005 no município de Santa Fé do Sul, permitindo depreender que houve um grande equívoco em sua divulgação pelo MEC, ao deixar de reconhecer as fragilidades na consolidação dos dados. Outro ponto interessante diz respeito à confirmação da existência de contraditórios e insustentáveis rankings do IDEB, amplamente divulgados pela mídia.

Palavras-chave: Avaliação Externa. Índice de Desenvolvimento da Educação Básica. Qualidade da Educação.

\section{CONTRIBUTIONS TO THE PUBLIC DEBATE ABOUT THE SCHOOL: THE INDEX OF BASIC EDUCATION DEVELOPMENT (IDEB)}

\begin{abstract}
This study presents some results obtained in the master research entitled "The Index of Basic Education Development (IDEB): a study of the city of Santa Fé do Sul / SP." It aims to address the operation of the Development Plan for Education (PDE) and its culmination in IDEB, globally analyzing its fundamentals, problems and prospects. It also aims, leaving data from desk research, interviews and questionnaires, reflect on the impact of rankings produced according to IDEB and practices increasingly currents of school accountability. To carry out the research in Santa Fe South had to elucidate mechanisms which triggered high IDEB increase in 2007 compared to 2005. The analyzes undertaken led to the finding of apparent failures in the consolidation of IDEB 2005 in the municipality of Santa Fe South, allowing infer that there was a great mistake in its disclosure by the MEC, by failing to recognize the weaknesses in data consolidation. Another interesting point concerns the confirmation of the existence of contradictory and unsustainable rankings IDEB, widely reported by the media.
\end{abstract}

Keywords: External Evaluation. Index of Basic Education Development. Quality of Education. 


\section{INTRODUÇÃO}

Diante das diversas transformações ocorridas no cenário mundial nas últimas décadas, fomentadas pelo processo da globalização, reformas educacionais e novas tecnologias, os sistemas educacionais têm passado por constantes mudanças na gestão e implementação de indicadores de qualidade baseados nas avaliações externas.

Partindo dessa constatação, este trabalho baseia-se em parte dos resultados obtidos na pesquisa de mestrado intitulada "O Índice de Desenvolvimento da Educação Básica (IDEB): um estudo do município de Santa Fé do Sul/SP" e tem como objetivo abordar a operacionalização do Plano de Desenvolvimento da Educação (PDE) e sua culminância no IDEB, analisando globalmente seus fundamentos, problemas $\mathrm{e}$ perspectivas. Pretende ainda, refletir sobre os impactos dos rankings produzidos em função do IDEB, bem como as práticas cada vez mais correntes de responsabilização da escolas.

Para dar conta dessas análises, o texto está dividido em duas partes. Na primeira, são abordadas as políticas de implementação do Plano de Desenvolvimento da Educação (PDE) e criação do IDEB e as ações com vistas à responsabilização da escola. Na segunda parte do texto, fazendo uso de reflexões desenvolvidas por alguns autores e dos resultados provenientes da pesquisa empírica no município de Santa Fé do Sul/SP, são analisados os problemas estruturais e as perspectivas do IDEB em relação a uma efetiva e consistente avaliação da qualidade da educação.

\section{O IDEB E A RESPONSABILIZAÇÃO DA ESCOLA}

A urgência na criação de políticas públicas objetivando $\circ$ desenvolvimento econômico e social do país desencadeou diversas ações do governo Lula. A principal delas foi o lançamento do Programa de Aceleração do Crescimento (PAC), em 28 de janeiro de 2007, que engloba um conjunto de políticas econômicas, planejadas para os quatro anos seguintes, e que tem como objetivo acelerar o crescimento econômico do Brasil, sendo uma de suas prioridades o investimento em infraestrutura, saneamento, habitação, transporte, energia e recursos hídricos, entre outros.

Frente aos objetivos do PAC, cada ministério teve que apresentar uma série de ações que se enquadrariam no referido programa. O Ministério da Educação aproveitou, então, e lançou em 24 de abril de 2007, o Plano de Desenvolvimento da Educação (PDE), lançado por meio do Decreto no 6.094. A partir da implantação do PDE novos rumos foram traçados em termos de políticas educacionais para se tentar alcançar níveis de qualidade de ensino mais elevados, principalmente com a criação do Índice de Desenvolvimento da Educação Básica (IDEB).

O IDEB foi criado em 2007 pelo INEP e resulta da combinação de dois fatores que interferem na qualidade da educação: a) indicadores de fluxo (taxas de aprovação, reprovação e evasão), medidos pelo Censo Escolar, e b) indicadores de desempenho em exames padronizados como o SAEB e Prova Brasil, realizados a cada dois anos ao final de determinada etapa da educação básica.

A Prova Brasil e o Sistema Nacional de Avaliação da Educação Básica (SAEB), criados pelo INEP, são avaliações em larga escala, cujos testes são aplicados na quarta e oitava séries (quinto e nono anos) do ensino fundamental e terceira série do ensino médio e estão restritos a aplicação de testes de Língua Portuguesa, Matemática e questionários socioeconômicos. ${ }^{1}$

\footnotetext{
${ }^{1}$ As médias de desempenho utilizadas são as da Prova Brasil (para Idebs de escolas e municípios) e do Saeb (no caso dos Idebs dos estados e nacional). A Prova Brasil é realizada por
} 
Convém, por oportuno, ressaltar que a combinação de indicadores de fluxo e de proficiência dos alunos, que resultam no IDEB, é calculada em valores de 0 a 10. A meta do MEC é que o Brasil atinja até 2021, ano que antecede a comemoração do bicentenário da Independência do Brasil, níveis educacionais de países desenvolvidos, o que corresponde à média 6,0 para os anos iniciais do Ensino Fundamental. Importa evidenciar que o ponto de partida para a criação das metas foi a definição do IDEB 2005 (inicial), resultante dos dados de desempenho apresentados na Prova Brasil e dos Censos Escolares de 2005 e 2006, que permitiram a conjunção das taxas de aprovação.

$$
\text { Para Fernandes } 2 \text { (2007b, p.3) }
$$

"tecnicamente, para projetar as trajetórias esperadas para o IDEB ao longo do tempo, tanto para o Brasil como para os demais níveis de abrangência, parte-se do princípio de que essas trajetórias têm o comportamento de uma função logística". Nessa perspectiva, foram projetadas as seguintes metas para os sistemas de ensino brasileiros:

Tabela 1. O IDEB e as Projeções para o Brasil

\begin{tabular}{|c|c|c|c|c|c|c|}
\hline \multirow{2}{*}{} & \multicolumn{4}{|c|}{ Anos Iniciais do Ensino Fundamental } \\
\cline { 2 - 7 } & IDEB Observado & \multicolumn{4}{c|}{ Metas } \\
\cline { 2 - 7 } & $\mathbf{2 0 0 5}$ & $\mathbf{2 0 0 7}$ & $\mathbf{2 0 0 9}$ & $\mathbf{2 0 0 7}$ & $\mathbf{2 0 0 9}$ & $\mathbf{2 0 2 1}$ \\
\hline TOTAL & $\mathbf{3 , 8}$ & $\mathbf{4 , 2}$ & $\mathbf{4 , 6}$ & $\mathbf{3 , 9}$ & $\mathbf{4 , 2}$ & $\mathbf{6 , 0}$ \\
\hline Pública & 3,6 & $\mathbf{4 , 0}$ & $\mathbf{4 , 4}$ & 3,6 & 4,0 & 5,8 \\
\hline Estadual & 3,9 & $\mathbf{4 , 3}$ & $\mathbf{4 , 9}$ & 4,0 & 4,3 & 6,1 \\
\hline Municipal & 3,4 & $\mathbf{4 , 0}$ & $\mathbf{4 , 4}$ & 3,5 & 3,8 & 5,7 \\
\hline Privada & 5,9 & $\mathbf{6 , 0}$ & $\mathbf{6 , 4}$ & 6,0 & 6,3 & 7,5 \\
\hline
\end{tabular}

Fonte: INEP/MEC (2010).

Partindo da observação da tabela e de acordo com o MEC, no ano de 2005, primeiro ano

todos os alunos da etapa avaliada, enquanto o Saeb é realizado por amostragem.

${ }^{2}$ Idealizador do IDEB junto ao MEC. aferido pelo IDEB, o Brasil alcançou apenas 3,8 pontos na escala para os anos iniciais do ensino fundamental, supondo-se, de acordo com as metas estabelecidas, a existência de uma precária situação educacional.

Muito embora o IDEB correspondente ao ano de 2007 e 2009 aponte que o Brasil subiu para a média de 4,2 e 4,6 pontos respectivamente, pontuações acima das metas previstas, estes índices ainda estão muito distantes dos 6,0 pontos, que é a média que os países desenvolvidos da Organização para a Cooperação e Desenvolvimento Econômico (OCDE) têm nessa escala ${ }^{3}$.

Levando-se em conta 0 que foi observado, a lógica do Ministério da Educação para que o Brasil chegue à média 6,0 em 2021, período estipulado tendo como base o já citado bicentenário da Independência em 2022, é que cada sistema evolua segundo pontos de partida distintos, e com esforço maior daqueles que partem em pior situação, tendo como objetivo explícito a redução da desigualdade educacional.

No entanto, conforme sustenta Fernandes (2007b, p. 4-5), a meta maior do IDEB é mais ambiciosa que os 6,0 pontos em 2021, pois ao se referir ao esforço dos sistemas de ensino, afirma que se for seguida a logística projetada "é garantido que a desigualdade entre eles se reduza até que, em $\lambda$ (2096), todos tenham o mesmo valor de IDEB $(9,9)$ ". O gráfico a seguir exemplifica a trajetória do IDEB (considerando a 1 a fase do EF) para o Brasil e dois Estados diferentes, até que seja alcançada a meta de convergência.

\footnotetext{
${ }^{3}$ A comparação da média brasileira no IDEB com os índices internacionais foi possível devido a uma técnica de compatibilização entre a distribuição das proficiências observadas no PISA (Programme for Internacional Student Assessment) e no SAEB.
} 


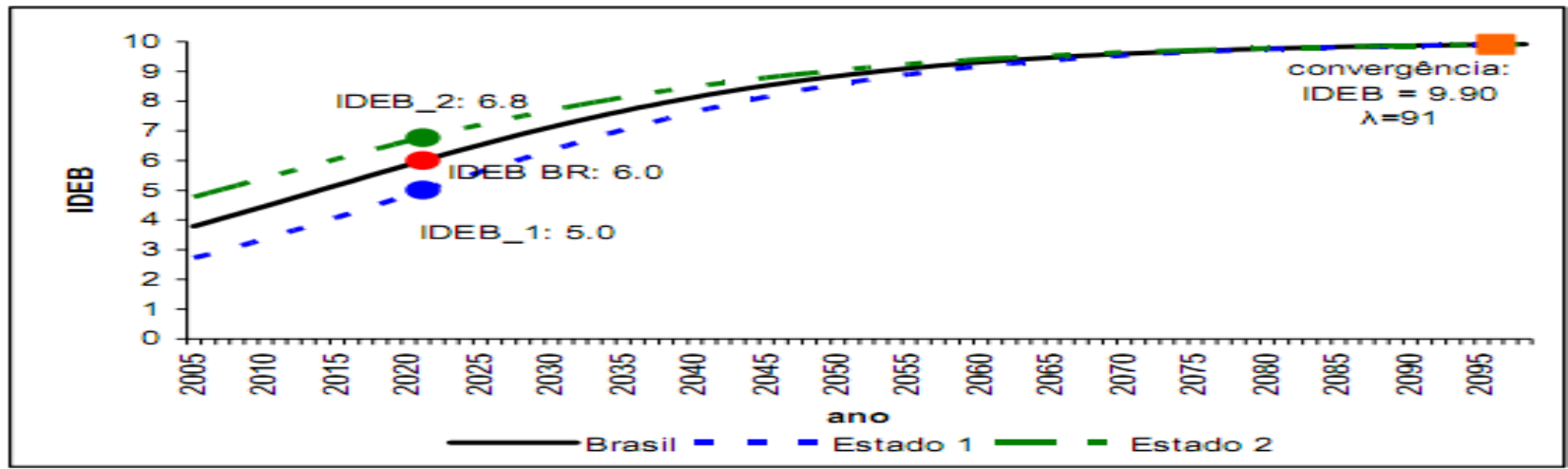

Figura 1. Comportamento Esperado do IDEB.

Fonte: INEP/MEC (2010)

Segundo o gráfico, é possível observar que em 2021, ano em que o Brasil atinge a meta de 6,0, O Estado 1 apresenta IDEB 5,0 e no Estado 2 o IDEB é 6,8. O que fica claro é o fato de que "cada município e Estado despenderá um esforço diferente para que em 2096 (após 91 anos) a desigualdade apresentada pelo IDEB desapareça". (FERNANDES, 2007b, p. 5, grifos nossos).

Diante desse quadro, duras críticas têm sido tecidas contra esse tipo de política de avaliação visto que desconsideram que parte dos problemas educacionais advém do próprio formato do projeto ideológico neoliberal, no qual se reduz a qualidade à igualdade de acesso às escolas, mas, em contrapartida, se estimula a competição e o ranqueamento entre elas, gerando espaços para ricos e espaços para pobres, segregando ainda mais as camadas populares no interior dos sistemas de ensino.

[...] A "responsabilização" gerará escolas que concentrarão exclusivamente pobres, onde 0 IDEB não importa, pois "já se sabe que não dá para esperar muito desses alunos". No caso da rede, se um subconjunto de escolas elevar o índice, e este atingir a pontuação combinada entre prefeito e governo federal, já é suficiente; as demais continuarão onde estão provavelmente estas serão as que atenderão à pobreza, que é guardada na escola para não incomodar, mesmo que não aprenda. No caso da escola não será diferente, muda apenas a unidade de análise. [...] (FREITAS, 2007, p. 976)

Dessa forma, tendo em vista outras ideias já discutidas por Freitas (2007) podemos levantar as seguintes questões complementares para entender as dimensões dos fenômenos desencadeados pelo IDEB: 1) As escolas vão querer a pobreza dentro dela, com o risco de ver seu IDEB piorar? 2) Mais ainda, o que fazer com escolas em que a evasão e a repetência não se dão por causas pedagógicas, responsabilizar gestores e docentes, mesmo assim?

Aliado a tudo isso ainda há o risco da liberação descontrolada do fluxo escolar, a introdução irreflexiva de quaisquer assessorias privadas nos sistemas de ensino e, inclusive, o treino contínuo de versões anteriores da Prova Brasil ou SAEB na ânsia da busca por resultados, tudo isso para evitar que os índices caíam e ocorra a responsabilização unilateral dos profissionais da escola. Pior ainda, é acreditar fielmente que uma média elevada no IDEB represente uma educação de qualidade, isto porque, um índice alto pode não significar melhoria para todos os alunos, já que se um 
determinado número de alunos for bem, a média subirá, mesmo que muitos outros continuem com baixíssimo nível de aprendizagem escolar.

Obviamente que isso não significa que as escolas não tenham que ser, verdadeiramente, instâncias eficazes de construção de uma aprendizagem sólida, mas, também, não podem ser reduzidas a meros reprodutores de ideologias mercadológicas. Em consonância com essas ideias muitos autores concordam que é importante saber se a aprendizagem em uma escola de periferia é baixa ou alta, mas

[...] fazer do resultado o ponto de partida para um processo de responsabilização da escola via prefeituras leva-nos a explicar a diferença baseados na ótica meritocrática liberal: mérito do diretor que é bem organizado; mérito das crianças que são esforçadas; mérito dos professores que são aplicados; mérito do prefeito que deve ser reeleito etc. Mas e as condições de vida dos alunos e professores? $\mathrm{E}$ as políticas governamentais inadequadas? O que dizer da permanente remoção de professores e especialistas a qualquer tempo, pulando de escola em escola? [...] O que dizer dos alunos que habitam as crescentes favelas sem condições mínimas de sobrevivência e muito menos para criar um ambiente propício ao estudo? Sem falar do número de alunos em sala de aula. (FREITAS, 2007, p. 970, grifos nossos).

Portanto, devemos insistir que não acolhemos a ideia de que deve haver a crítica pela crítica, quando se aborda as vertentes atreladas à avaliação externa, mas, sobretudo, que é pela forma como se dá o seu uso, dentro da ideologia em que se respalda, que ela se torna instrumento, muitas vezes, com fins pouco efetivos para a melhoria da qualidade da educação brasileira, ainda mais se pensarmos na existência de regiões onde há desde professores leigos a desvio de recursos financeiros, altos níveis de pobreza e desvalorização do magistério, ou seja, problemas antes de tudo governamentais do que educacionais.

\section{OS PROBLEMAS ESTRUTURAIS E ALGUMAS PERSPECTIVAS SOBRE O IDEB}

Os dados publicados pelo INEP referentes às metas do IDEB para 2007 e 2009 apresentaram resultados positivos incipientes, na análise do Ministério da Educação e de muitos órgãos da mídia.

Em relação ao desempenho dos municípios, conforme divulgado ${ }^{4}$, em 2007 apenas 64 dos mais de 5 mil municípios brasileiros tinham para turmas de $1^{\underline{a}}$ a $4^{\underline{a}}$ série um ensino público com qualidade de países desenvolvidos, o que significa ter nota 6,0 na escala de 0 a 10 do IDEB.

Em contraposição a esse cenário, os municípios de Adolfo e Santa Fé do Sul, ambos do Estado de São Paulo, encontravam-se no topo da escala nacional da qualidade da educação, segundo dados do INEP referentes a 2007. município de Santa Fé do Sul, por exemplo, alcançou no IDEB uma média excepcional de 7,6 pontos. Este município, bem como o de Adolfo, saiu em apenas 2 anos, de uma média de 4,7 pontos para os elevados índices observados, que, se enquadrados nas normas da OCDE, equivalem a de países desenvolvidos de "notável qualidade educacional".

No entanto, nesse contexto, o maior destaque fica por conta da Escola Municipal "Elisabeth Maria Cavaretto de Almeida", da cidade de Santa Fé do Sul, considerada a melhor escola do Brasil, com média de 8,6 pontos no IDEB 2007. Convém evidenciar que o resultado do IDEB 2005 indicou que esta escola havia

\footnotetext{
${ }^{44_{4}}$ Jornal "O Estado de São Paulo", 23 jun. 2008, s/p.
} 
obtido uma média de apenas 2,6 pontos. Se for empreendida uma comparação em termos percentuais, podemos constatar um crescimento de aproximadamente $230 \%$ no período ou, que o índice analisado mais que triplicou. $\mathrm{Na}$ tabela seguinte é possível notar o grande avanço obtido

Tabela 2. IDEB 2005 e 2007 - Santa Fé do Sul - SP

\begin{tabular}{|l|l|l|l|}
\hline \multicolumn{1}{|c|}{ ESCOIa - } & $2005-$ & $2007=$ \\
\hline BAIRRO DA BELA VISTA EM & 5.7 & & Meta 2007 \\
\hline BENEDICTO DE LIMA PROF EM & 5.3 & 7.6 & 5.8 \\
\hline MARINA DE OLNEIRA PROFA EM & 5.6 & 5.1 & 5.4 \\
\hline CIRLEY VOLPE LOPES PROFA EM & 5.7 & 7.9 & 5.6 \\
\hline ELISABETH MARIA CAVARETTO DE ALMEIDA PROFA EM & 2.6 & 8.6 & 2.9 \\
\hline ROSIMARES CAMARGO BENITEZ EM & 5.3 & 7.7 & 5.4 \\
\hline
\end{tabular}

Fonte: MEC/INEP (2010)

Em relação a esse tipo de avanço demasiadamente elevado para tão pouco tempo diversas controvérsias foram levantadas, inclusive, sobre 0 fato de talvez não corresponderem, necessariamente, a um aumento real dos fatores de desempenho dos alunos e aumento das taxas de aprovação. Como exemplo, tomamos um estudo de Camargo, Pinto e Guimarães (2008, p. 831), no qual enfatizam que se constatou que, praticamente, "mais da metade (668 municípios) apresentou uma variação positiva do IDEB na comparação igual ou maior que 30\% entre 2005 e 2007; 287 (23\%) tiveram uma variação igual ou maior a 40\%; 167 $(13,5 \%)$ viram seu IDEB crescer $50 \%$ ou mais; 18 municípios tiveram 0 índice dobrado e 4 triplicados." Sobre este fato, os autores depreendem que:

[...] qualquer pesquisador ou gestor educacional sabe que as redes de ensino não apresentam saltos desta natureza em prazo tão curto. Quando analisamos os municípios que apresentaram crescimento igual ou acima de $50 \%$ constatamos que a variação decorreu principalmente da variação positiva na taxa de aprovação, um dos dois componentes do tanto pela escola em questão, quanto em relação a quatro outras escolas do mesmo município que também tiveram avanços da ordem de $35 \%$ de crescimento em média, em cerca de dois anos. 
Camargo Pinto e Guimarães (2008, p. 832), ao abordar duas outras problemáticas estruturais a respeito do IDEB, ressaltam que:

Falta ao IDEB uma dimensão que leve em conta os insumos educacionais (qualificação dos professores, recursos colocados à disposição dos alunos etc.). Da maneira como o índice está montado, ao invés de investir em medidas de longo prazo que sabidamente melhoram a qualidade do ensino, os municípios, para não verem minguar o repasse de recursos do governo federal, tendem a adotar medidas cosméticas, ou mesmo a valer-se de fraude. A imprensa noticiou casos de escolas que diziam a determinados alunos, aqueles que tinham as maiores dificuldades, que não precisariam vir à escola no dia da Prova Brasil, por exemplo. O outro grande problema do IDEB é que a sua lógica leva inevitavelmente ao estabelecimento de ranking entre municípios e entre escolas. Ao contrário do que acontece em outros países onde os resultados dos testes são ponderados levando-se em conta o perfil socioeconômico dos alunos e o tamanho das escolas (dois fatores com forte influência nos resultados) 0 MEC divulga simplesmente as notas sem esse cuidado mínimo.

Dado o exposto, aí estão, portanto, outros fatores estruturais muito criticados pelos autores da atualidade. Araújo (2007, apud FREITAS, 2007) conclui que o ranqueamento entre as redes de ensino, para acirrar a competição e para pressionar, via opinião pública, o alcance de melhores resultados mantém uma lógica perversa. Isto pode ser comprovado pela fuga das escolas com menores notas dos professores mais experientes e qualificados e dos alunos cujos pais apresentam maior envolvimento e possuem mais recursos, reforçando a desigualdade do sistema educacional.

Posto isto, também nos cumpre ressaltar que alcançar o acesso com permanência e sucesso na escola, sob à lógica de políticas de avaliação como o IDEB se torna um grande problema, pois ora os sistemas de ensino considerados os melhores do Brasil devem ser seguidos como modelos para uma educação de qualidade, conforme divulgam o MEC e a mídia, ora são esquecidos, pois acabam substituídos por novos campeões no ranking do desempenho escolar. Enfatizamos isto porque se verificarmos os dados da tabela a seguir, veremos a inconsistência da manutenção dos resultados obtidos de um biênio para outro. No caso específico de Santa Fé do Sul, o município já não se situa mais entre os quinze melhores do Brasil, tendo uma queda acentuada no IDEB de 2007 para 2009, assim como a maioria das demais cidades. 
Tabela 3. Os Melhores Municípios no IDEB 2007 e no IDEB 2009

\begin{tabular}{|c|c|c|c|c|}
\hline \multicolumn{5}{|c|}{ Ensino Fundamental Regular - Séries Iniciais (Até a 4ª série) } \\
\hline UF & Nome do Município & Rede & IDEB 2007 & IDEB 2009 \\
\hline SP & ADOLFO & Pública & 7,7 & $7,0(-\mathbf{0 , 7})$ \\
\hline SP & SANTA FÉ DO SUL & Pública & 7,6 & $6,0(-1,6)$ \\
\hline SP & COSMORAMA & Pública & 7,5 & $6,7(-0,8)$ \\
\hline RS & CENTENÁRIO & Pública & 7,3 & $--^{\star}$ \\
\hline SP & SANTA RITA D'OESTE & Pública & 7,2 & $5,5(-1,7)$ \\
\hline$\overline{\mathrm{SP}}$ & |TAQUARIVAÍ & Pública & $7,7,2$ & $5,4(-1,8)$ \\
\hline SP & CAJURU & Pública & 7,0 & $8,6(+1,6)$ \\
\hline SP & TURMALINA & Pública & 7,0 & $6,9(-0,1)$ \\
\hline SP & BARRA DO CHAPÉU & Pública & 6,9 & $5,4(-1,5)$ \\
\hline $\mathrm{SP}$ & TERRA ROXA & Pública & 6,9 & $5,8(-1,1)$ \\
\hline SP & VALENTIM GENTIL & Pública & 6,9 & $5,7(-1,2)$ \\
\hline SP & VALPARAÍSO & Pública & 6,9 & $5,2(-1,7)$ \\
\hline SP & ESTRELA D'OESTE & Pública & 6,8 & $6,0(-0,8)$ \\
\hline$\overline{\mathrm{SP}}$ & LUCIANÓPOLIS & Pública & 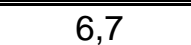 & $5,9(-0,8)$ \\
\hline MG & $\begin{array}{l}\text { SÃO JOÃO BATISTA DA } \\
\text { GLÓRIA }\end{array}$ & Pública & 6,7 & $7,0(+0,3)$ \\
\hline
\end{tabular}

Fonte: MEC/INEP (2010)

* Não informado pelo MEC/INEP.

Devemos observar que 0 efeito efêmero dos resultados do IDEB que ora são comemorados, ora são esquecidos, tem permeado os rankings criados desde 2005. A cada novo biênio um conjunto de municípios emerge "milagrosamente" como campeão da qualidade na educação. Depois, como já comentamos, é esquecido, ao ser substituído por um novo grupo de "cidades modelos", fato que mostra a fragilidade do índice ao não conseguir captar fidedignamente os processos educativos referentes à aprendizagem escolar, gerando contradições, equívocos e falhas técnicas, como ocorreu em Santa Fé do Sul em 2005.

Sobre estes fatos, uma gestora escolar de Santa Fé do Sul faz questão de enfatizar que a publicização do aumento expressivo do IDEB 2005 (2,6 pontos) para o IDEB 2007 (8,6 pontos), na Escola Municipal "Profa Elisabeth Maria Cavaretto de Almeida é um grande equívoco, porque se chegou "[...] à conclusão que esses 2,6 não existiram diante da conjuntura de que todas as escolas na época e hoje também trabalham juntas numa mesma linha de pensamento, num mesmo tudo, e que as outras escolas estavam com 5,9 e essa escola a única com 2,6".

No mesmo vértice, uma das gestoras da Secretaria Municipal de Educação que atuou no período, explica que ao observar as notas das disciplinas de Língua Portuguesa e Matemática de todas as escolas municipais, constatou-se que todas estavam no mesmo patamar. Entretanto, 
esclarece que fazia um ano da implantação do ensino fundamental de nove anos e os dados divulgados pelo INEP apontavam diversas retenções nos $1^{\circ}, 2^{\circ}$ e $4^{\circ}$ anos, o que não era possível, pois a retenção ocorria apenas nos $3^{\circ} \mathrm{e}$ $5^{\circ}$ anos (subciclos).

[...] no censo escolar de 2005, o $1^{\circ}$ ano da escola "Elisabeth" constava com 58 alunos. Em dezembro de 2005, aparecia no INEP a mesma série com 136 alunos. Ou seja, de março a dezembro nós recebemos praticamente 80 alunos. Quase que 200 por cento. $E$ isso também aparecia nas séries seguintes, nos $2^{\circ}$, $3^{\circ}$, $4^{\circ}$ e $5^{\circ}$ anos. Nós pedimos também que o INEP nos informasse, extraoficial, a questão das retenções. $E$ o INEP nos informou que numa somatória total a escola apresentava 76 por cento de retenção. $E$ nós questionamos, porque se você puxar pelo rendimento escolar da escola em 2006 que é referente a 2005, uma média de 5 alunos foram retidos na unidade escolar. Então ficava uma discrepância. Uma coisa irreal em relação aos dados oficiais que nós tínhamos no município, informados no censo escolar de 2005, com fechamento em 2006 e 0 que estava sendo apresentado pelo INEP. O INEP nos informou que o censo já estava fechado e que não havia nenhuma possibilidade de que esse censo fosse alterado. Nos informou também, através das letras oficiais, de que o que houve foi uma migração errada ou a chamada "sujeira de banco". [...] Então a gente não esconde esse erro de que houve uma... Um erro na migração de dados da escola "Elisabeth" no ano de 2005 entre a Secretaria de Estado da Educação e o INEP.
Em relação a este tipo de ocorrência, podemos verificar o que Camargo, Pinto e Guimarães, (2008) explicam sobre o fato de os resultados divulgados nos rankings, talvez não corresponderem, necessariamente, a um aumento real da qualidade da educação. Podemos constatar, ainda, que é legítima a indignação dos autores com os 18 municípios que tiveram o índice dobrado e 4 triplicados em 2007, pois, no que concerne ao município de Santa Fé do Sul, suas predições podem ser ratificadas tanto nos relatos dos profissionais da educação indagados como em documentos coletados. Para confirmarmos esta asserção é necessário tomar a fala da mesma gestora, que explica que o salto obtido no IDEB 2007 pode ser um efeito compensatório concedido pelo MEC para corrigir os erros de 2005.

Eu creio que sim. Porque como foi um dos primeiros anos que eles começaram a migrar os dados... Tanto que uma das dificuldades que 0 INEP encontrou para fazer os dados do IDEB, eram dados fidedignos. Por isso que 0 estado de São Paulo migrou o que a gente já realiza na PRODESP desde 95. Houve o primeiro ano de migração desses dados. Então eu creio que deve ter tido muitas falhas. Então agora tem se aperfeiçoado. E ele constatou uma realidade: que a escola não comporta 1000 alunos, que a escola não tem 75 por cento de retenção, que ela não tem 12,9 por cento de distorção idadesérie. E que a escola está no mesmo patamar das demais.

O IDEB 2009 divulgado em meados de 2010 demonstrou que o município estabilizou-se na média de 6,0 pontos, enquanto suas unidades escolares tiveram índices equilibrados.

Tabela 4. IDEB das Escolas Municipais de $1^{\circ}$ a $5 \% / E F$ de Santa Fé do Sul - SP 


\begin{tabular}{|c|c|c|c|c|c|c|c|c|c|c|c|}
\hline \multirow[b]{2}{*}{ Escola $\uparrow$} & \multicolumn{3}{|c|}{ Ideb Observado } & \multicolumn{8}{|c|}{ Metas Projetadas } \\
\hline & $2005 *$ & $2007 \div$ & $2009 *$ & $2007 *$ & $2009 *$ & $2011 *$ & $2013 \div$ & $2015 *$ & $2017 \div$ & $2019 *$ & $2021 *$ \\
\hline BAIRRO DA BELA VISTA EM & 5.7 & & & 5.8 & 6.1 & 6.4 & 6.6 & 6.8 & 7.0 & 7.2 & 7.4 \\
\hline BENEDICTO DE LIMA PROF EM & 5.3 & 7.6 & 5.3 & 5.4 & 5.7 & 6.0 & 6.3 & 6.5 & 6.7 & 6.9 & 7.1 \\
\hline CIRLEY VOLPE LOPES PROFA EM & 5.7 & 7.9 & 6.0 & 5.8 & 6.1 & 6.4 & 6.6 & 6.8 & 7.0 & 7.2 & 7.4 \\
\hline ELISABETH MARIA CAVARETTO DE ALMEIDA PROFA EM & 2.6 & 8.6 & 6.1 & 2.9 & 3.6 & 4.2 & 4.5 & 4.8 & 5.1 & 5.3 & 5.6 \\
\hline MARINA DE OLIVEIRA PROFA EM & 5.6 & 6.1 & 5.7 & 5.6 & 5.9 & 6.3 & 6.5 & 6.7 & 6.9 & 7.1 & 7.3 \\
\hline ROSIMARES CAMARGO BENTEZ EM & 5.3 & 7.7 & 6.5 & 5.4 & 5.7 & 6.1 & 6.3 & 6.5 & 6.7 & 7.0 & 7.2 \\
\hline
\end{tabular}

Fonte: MEC/INEP (2010)

Aduzimos pelos dados apresentados que o mecanismo que resulta no IDEB tem se mostrado bastante incipiente e questionável. $\mathrm{A}$ fragilidade do INEP e da Secretaria de Educação do Estado de São Paulo para consolidar e transferir as informações sobre aprovação, retenção e evasão escolar causou, segundo dados obtidos na cidade de Santa Fé do Sul, grande transtorno e perplexidade frente aos resultados amplamente divulgados na mídia em forma de rankings.

\section{CONSIDERAÇÕES FINAIS}

Perpassamos a discussão, acrescentando dados coletados com gestores e fontes documentais por ocasião da pesquisa de mestrado intitulada "O Índice de Desenvolvimento da Educação Básica (IDEB): um estudo do município de Santa Fé do Sul/SP". Diante dos dados apresentados, entendemos que ao analisar - IDEB e seus resultados, torna-se inapropriado reduzir toda a complexidade dos contextos educacionais a uma política de avaliação que pretenda considerar o processo educativo como produto e não como processo.

Ademais, verificamos que os resultados do IDEB têm se mostrado ainda incipientes e questionáveis, ao ponto que é possível observar uma constante substituição de "cidades-modelos", divulgadas nos rankings, a cada novo biênio. A falha do INEP e da Secretaria de Educação do Estado de São Paulo na transferência dos dados sobre aprovação, retenção e evasão escolar, ratifica que a escola Elisabeth Maria Cavaretto de Almeida, embora divulgado pela mídia e pelo MEC, nunca teve em 2005 o IDEB de 2,6 pontos.

\section{REFERÊNCIAS}

BRASIL. Ministério da Educação. Fundo Nacional de Desenvolvimento da Educação. Plano de Desenvolvimento da Educação. Plano de Metas Compromisso Todos pela Educação. Guia de programas. Brasília, DF, 2007.

Ministério da Educação. Decreto Presidencial $n^{\circ}$ 6.094, de 24 de abril de 2007. Dispõe sobre a implementação do Plano de Dispõe sobre a implementação do Plano de Metas Compromisso Todos pela Educação. Diário Oficial da União, Brasília, DF, 25 abr. 2007.

. Ministério da Educação e Cultura. IDEB: como melhorar os índices. Disponível em: $<$ http://portal.mec.gov.br>. Acesso em: 18 jun. 2008.

. Ministério da Educação e Cultura. Taxa de Aprovação, Prova Brasil, IDEB e Projeções por Município e Rede. Disponível em: <http://www.inep.gov.br/download//deb/Resultado/ republicacao/Divulgacao_4serie_Municipios.xls>. Acesso em: 20 jun. 2009.

Programa de Aceleração do Disponivel em: <http://www.brasil.gov.br/pac/>. Acesso em: 17 jun. 2009.

CAMARGO, R. B. de; PINTO, J. M. de R.; GUIMARAES, J. L. Sobre o financiamento no Plano de Desenvolvimento da Educação. Cadernos de Pesquisa, São Paulo, v. 38, n. 135, dez. 2008.

FERNANDES, R. Índice de Desenvolvimento da Educação Básica (IDEB). Instituto Nacional 
de Estudos e Pesquisas Educacionais Anísio Teixeira, Brasília, 26 p. 2007a.

\section{Índice de Desenvolvimento da}

Educação Básica (IDEB): metas intermediárias para a sua trajetória no Brasil, estados, municípios e escolas. Instituto Nacional de Estudos e Pesquisas Educacionais Anísio Teixeira, Brasília, 5 p. 2007b.

FREITAS, L. C. Eliminação adiada: o ocaso das classes populares no interior da escola e a ocultação da (má) qualidade do ensino. Educação \& Sociedade, Campinas, v. 28, n. 100, p. 965-987, out. 2007. http://dx.doi.org/10.1590/S0101$\underline{73302007000300016}$

IDEB: apesar do avanço, só 64 cidades têm nota de países desenvolvidos. O Estado de São Paulo, São Paulo, 23 jun. 2008. 